\title{
Improvement of the bioluminescence reporter system for real-time monitoring of circadian rhythms in the cyanobacterium Synechocystis sp. strain PCC 6803
}

\author{
Ken-ichi Kucho ${ }^{1}$, Kazuhiro Aoki ${ }^{2, \dagger}$, Shigeru Itoh $^{2}$ and Masahiro Ishiura ${ }^{1,3,4, *}$ \\ ${ }^{1}$ Center for Gene Research, Nagoya University, Furo-cho, Chikusa-ku, Nagoya, 464-8602, Japan \\ ${ }^{2}$ Division of Material Science, Graduate School of Science, Nagoya University, Furo, \\ Chikusa-ku, Nagoya 464-8602, Japan \\ ${ }^{3}$ Division of Biological Science, Graduate School of Science, Nagoya University, \\ Furo-cho, Chikusa-ku, Nagoya 464-8602, Japan \\ ${ }^{4}$ Bio-oriented Technology Research Advancement Institution (BRAIN), \\ Nagoya University, Furo-cho, Chikusa-ku, \\ Nagoya 464-8602, Japan
}

(Received 9 December 2004, accepted 25 January 2005)

\begin{abstract}
Circadian rhythm is a self-sustaining oscillation whose period length coincides with the 24-hour day-night cycle. A powerful tool for circadian clock research is the real-time automated bioluminescence monitoring system in which a promoter region of a clock-controlled gene is fused to a luciferase reporter gene and rhythmic regulation of the promoter activity is monitored as bioluminescence. In the present study, we greatly improved the bioluminescence reporter system in the cyanobacterium Synechocystis sp. strain PCC 6803. We fused an 805-bp promoter region of the dnaK gene seamlessly to the $l u x A$ coding sequence and integrated the $\mathrm{P}_{d n a K}:: \operatorname{lux} A B$ fusion gene into a specific intergenic region of the Synechocystis genome (targeting site 1). The resulting new reporter strain, PdnaK::luxAB(-), showed 12 times the bioluminescence intensity of the standard reporter strain, CFC2. Furthermore, we generated strain PdnaK::luxAB(+), in which the $\mathrm{P}_{\text {dnaK }}:$ lux $A B$ fusion gene and the selection-marker spectinomycin resistance gene are transcribed in opposite directions. The PdnaK::luxAB $(+)$ strain showed 19 times the bioluminescence intensity of strain CFC2. The procedures used to increase the bioluminescence intensity are especially useful for bioluminescence monitoring of genes with low promoter activity. In addition, these reporter constructs facilitate bioluminescence monitoring of any gene because the promoter fragments they contain can easily be replaced by digestion with unique restriction enzymes. They would therefore contribute to a genome-wide analysis of gene expression in Synechocystis.
\end{abstract}

Key words: bioluminescence, circadian rhythm, dnaK, luxAB, Synechocystis

\section{INTRODUCTION}

Circadian rhythm is a self-sustaining oscillation whose period length coincides with the 24-hour day-night cycle. Many biological activities show circadian patterns, allowing organisms to adapt to daily fluctuations in the environment. Circadian rhythms are widespread and involved in functions as diverse as human sleep-wake

Edited by Kazuhiro Kutsukake

* Corresponding author. E-mail: ishiura@gene.nagoya-u.ac.jp

† Present address: Department of Tumor Virology, Research Institute for Microbial Diseases, Osaka University, Yamadaoka, Suita-shi, Osaka 565-0871, Japan cycles and cyanobacterial nitrogen fixation. Endogenous oscillators that generate circadian rhythms are called circadian clocks. Cyanobacteria are the only bacterial species found to have circadian clock. Three clock genes kaiA, kaiB, and kaiC - have been identified in Synechococcus sp. strain PCC 7942, and positive and negative feedback regulations of the kai genes are proposed to be the core mechanism generating the circadian oscillation (Ishiura et al., 1998).

Real-time automated bioluminescence monitoring is a powerful tool for circadian clock research since it allows high resolution, sustained measurements of circadian rhythms (Aoki et al., 1995; Kondo et al., 1993; Millar et 

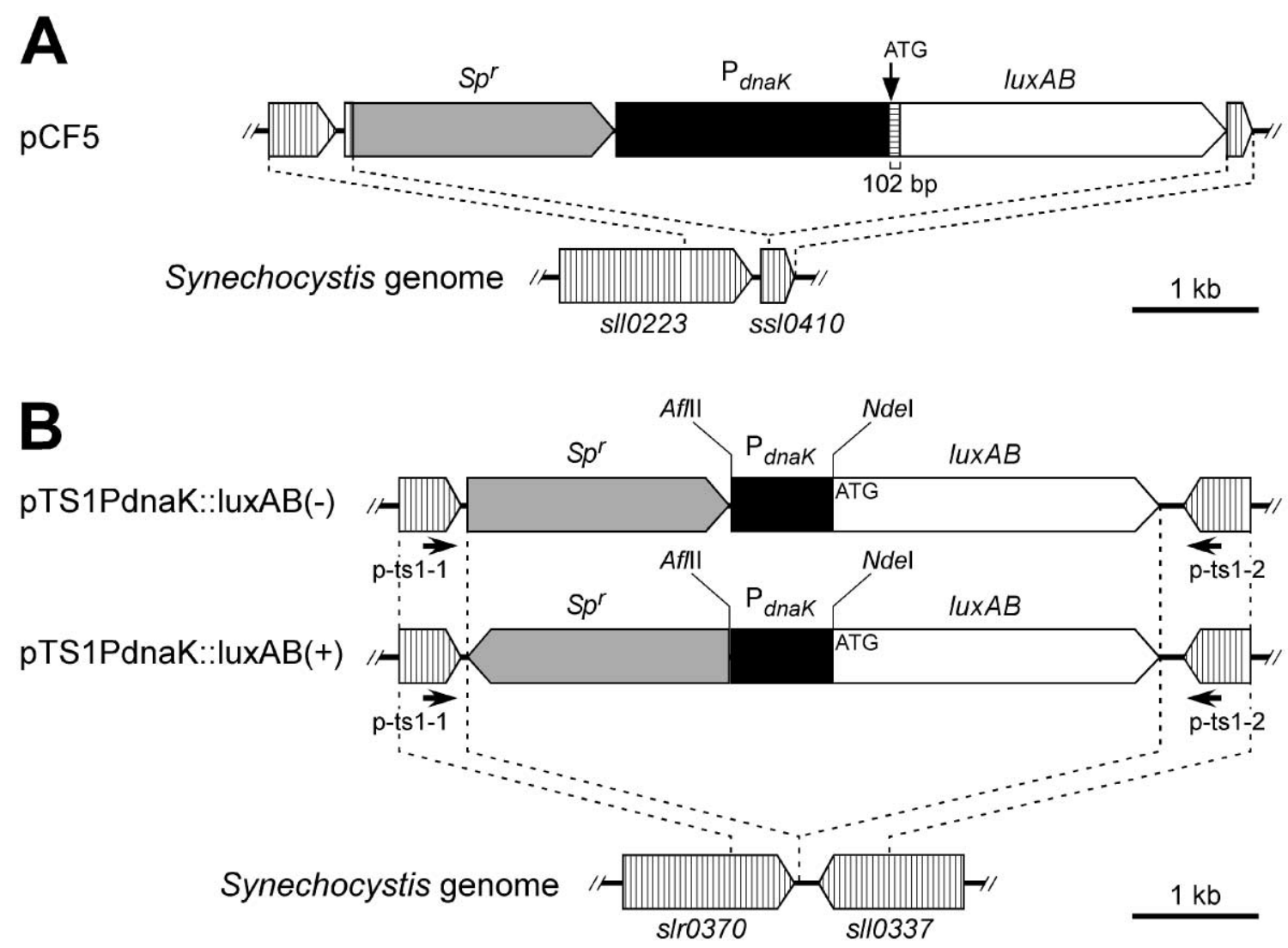

Fig. 1. Schematic representation of the bioluminescence reporter constructs and the integration sites in the Synechocystis genome. (A) pCF5, which was used to generate strain CFC2 (Aoki et al., 1995). (B) pTS1PdnaK::luxAB(-) and pTS1PdnaK::luxAB(+), which were used to generate strains PdnaK::luxAB(-) and PdnaK::luxAB(+), respectively. The boxes with an arrowhead indicate open reading frames (ORFs) and their transcription direction. Arrowheads designate PCR primers used for confirmation of the expected integration of the reporter constructs. ORF numbers are according to the CyanoBase (http://www.kazusa.or.jp/cyano/).

al., 1992; Onai et al., 2004). In the case of cyanobacteria, a promoter region of a clock-controlled gene is fused to bacterial luciferase gene $\operatorname{lux} A B$, and the resulting fusion gene is integrated into the genome (Aoki et al., 1995; Kondo et al., 1993; Onai et al., 2004). Then, rhythmic regulation of the promoter activity is continuously monitored as bioluminescence using $n$-decanal, a membranepermeating and volatile substrate without perturbing cells.

We previously demonstrated bioluminescence circadian rhythms in the cyanobacterium Synechocystis sp. strain PCC 6803 using a strain CFC2, which carries a luxAB bioluminescence reporter gene fused to a promoter region of the dnaK gene $\left(\mathrm{P}_{d n a K}\right)$ (Aoki et al., 1995). Because Synechocystis can carry out light-activated heterotrophic growth in glucose-supplemented medium in darkness (Anderson and McIntosh, 1991), the system is useful for elucidating the circadian regulatory mechanisms in darkness (Aoki et al., 1997). In addition, by performing DNA microarray analysis of clock-controlled genes, we identified many interesting genes implicated in circadian clock output mechanisms (Kucho et al., 2004). Despite very high activity of $d n a K$ promoter, however, bioluminescence intensity in strain CFC2 is low (approximately 1/20th that of the psbAI reporter strain of Synechococcus) (Aoki et al., 1995). This hampered further application of the bioluminescence reporter system to genes with weak promoter activity. Strain CFC2 had two known problems (Fig. 1A): (i) the 102-bp sequence that was inserted at the junction of the $\mathrm{P}_{d n a K}$ and $l u x A$ coding sequences added 34 extra amino acids to the amino-terminus of the LuxA protein, and (ii) the reporter construct interrupted the coding sequence of the ssl0410 gene. It seemed likely that the strain's low bioluminescence intensity was a result of these factors, especially factor (i). In the present study, we report a great improvement in the bioluminescence intensity in Synechocystis.

\section{MATERIALS AND METHODS}

Bacterial strains, culture conditions, and manipulation of DNA. A glucose-tolerant strain of Synechocystis sp. strain PCC 6803 was maintained in BG-11 liquid medium (Rippka et al., 1979) or on BG-11 agar 
medium containing $1 \mathrm{mM}$ sodium thiosulfate, $10 \mathrm{mM} \mathrm{N}$ tris(hydroxymethyl)methyl-2-aminoethanesulfonic acid (TES, pH 8.2), and 1.5\% Bacto Agar (Nippon BD, Tokyo, Japan) at $30^{\circ} \mathrm{C}$ under constant light (LL) from white fluorescent lamps at $67 \mu \mathrm{mol} \mathrm{m} \mathrm{m}^{-2} \mathrm{sec}^{-1}$. Transformants were selected and segregated on BG-11 agar medium or BG-11 liquid medium containing $40 \mu \mathrm{g} / \mathrm{ml}$ spectinomycin sulfate. Escherichia coli strains HB101 and DH5 $\alpha$ were maintained at $37^{\circ} \mathrm{C}$ in Luria-Bertani broth (LB) liquid medium or on LB agar medium containing $1.2 \%$ agar (Sambrook et al., 2001). DNA manipulation and sequencing were performed by standard methods (Sambrook et al., 2001).

Generation of reporter constructs and Synechocystis reporter strains. To generate a $\mathrm{P}_{\text {dnaK }}: l u x A B$ fusion gene, we amplified a $d n a K$ promoter segment (nucleotides -1 to -805 , with the A of the ATG translation initiation codon defined as +1 ) by PCR using Synechocystis genomic DNA as a template and the primer set PdnaK805F (5'TTCTTAAGGTGACCATCAAGCCGGTGCG-3'; the AflII site is underlined) and PdnaK805R (5'-TTCATATGTTTAATTAACTCCCGTTAAC-3'; the NdeI site is underlined, and the CAT sequence complementary to the ATG initiation codon of the dnaK gene is italicized). The amplified fragment was subcloned into pT7Blue-T (Novagen, Madison, WI, USA) to yield pT7PdnaK. We amplified a 2.1-kb fragment carrying the coding region of the Vibrio harveyi luxAB gene set (Baldwin et al., 1984) by PCR with plasmid pLAV1 (gift of T. O. Baldwin) as the template and the primer set Lux-F1M (5'-TCATATGAAATTTGGAAACTTCCTTC-3'; the NdeI site is underlined, and the ATG initiation codon of the luxA gene is italicized) and Lux-R1M (5'-TTCTAGATTACGAGTGGTATTTGACG-3'; the $X b a \mathrm{I}$ site is underlined), and subcloned into pT7Blue-T to yield pT7Blue-T/luxAB. We excised the $\operatorname{lux} A B$ gene segment from $\mathrm{pT} 7 \mathrm{Blue}-\mathrm{T} / \mathrm{lux} \mathrm{AB}$ as a 2.1-kb $N d e \mathrm{I}-\mathrm{Xba \textrm {I }}$ fragment and ligated it into the NdeI-XbaI site of pT7PdnaK, which is just downstream of $\mathrm{P}_{\text {dnaK }}$, yielding $\mathrm{pT} 7 \mathrm{PdnaK}:$ :luxAB.

To generate a targeting vector that allows integration of the $\mathrm{P}_{\text {dnaK }}:$ lux $A B$ fusion gene into the Synechocystis genome (targeting site 1) by homologous recombination, we amplified $0.5-\mathrm{kb}$ and $0.7-\mathrm{kb}$ segments that corresponded to the genomic regions of Synechocystis between positions 2,370,160 and 2,370,681 and between 2,370,682 and 2,371,369 (Kaneko et al., 1996) by PCR using primer sets Up68NS1U (5'-CAGCTGTCCCAGCCTCTCAACCAC3') and Up68NS1L (5'-AGATCTGAATAGAAGAGCGATAAT-3'; the BglII site is underlined), and Lo68NS1U (5'AGATCTAGTGTAGGCGGTAAAGTC-3'; the $B g l \mathrm{II}$ site is underlined) and Lo68NS1L (5'-CAGCTGTTGGTGGAAAGTTGGCTC-3'), respectively. The amplified fragments were digested with $B g l \mathrm{II}$ and ligated into pGEM-T (Promega, WI, USA) to yield pUL68TS1. For elimina- tion of a NdeI site of pUL68TS1, the plasmid was digested with NdeI, blunt-ended with the Klenow fragment, and self-ligated. The resulting plasmid, pUL68TS1 $1 \Delta$ Nde, was digested with $B g l I I$ and ligated with a doublestranded oligonucleotide containing BamHI, AflII, KpnI, and EcoRV restriction sites (d[pGATCGGATCCCTTAAGGGTACCGATATC]) to yield pUL68TS1 $\triangle$ NdeMCS. We inserted the spectinomycin resistance gene $\left(S p^{r}\right)$ (Prentki and Krisch, 1984) and the transcription terminator sequence of the $E$. coli $r r n B$ gene (Orosz et al., 1991) derived from pTrc99A (GenBank accession number M22744) into the BamHI and KpnI sites of pUL68TS1 $\triangle$ NdeMCS, respectively, yielding pUL68TS1 $\triangle$ NdeMCS $\Omega T$.

We excised the $\mathrm{P}_{\text {dnaK }}:$ lux $A B$ fusion gene from pT7PdnaK::luxAB as an $A f l I I-X b a I$ fragment and ligated it into the $A f l \mathrm{II}-\mathrm{X} b a \mathrm{I}$ site of pUL68TS1 $1 \Delta \mathrm{NdeMCS} \Omega \mathrm{T}$, which locates between $S p^{r}$ and the $r r n B$ transcription terminator, to generate pTS1PdnaK::luxAB(-) (Fig. 1B). To generate pTS1PdnaK::luxAB(+), we excised a $S p^{r}$-containing fragment from pTS1PdnaK::luxAB(-) by BamHI digestion and ligated it back into the same BamHI site. We selected a clone in which $S p^{r}$ and the $\mathrm{P}_{d n a K}$ ::lux $A B$ fusion gene were transcribed in opposite directions (Fig. 1B). We sequenced all the PCR-amplified fragments and all the junctions resulting from ligation reactions and confirmed that their nucleotide sequences were correct.

We transferred the DNA constructs into Synechocystis cells as previously described (Williams, 1988) and selected spectinomycin-resistant colonies. We confirmed by PCR that the reporter constructs were integrated into targeting site 1 using the genomic DNA as a template and the primer set p-ts1-1 (5'-TGGCTTTGGGCGGGAACTTG3') and p-ts1-2 (5'-GCCATATTTAACGGGACAGC-3') (Fig. 1B).

Assay of bioluminescence rhythms. Synechocystis cells were grown on BG-11 agar under LL at $30^{\circ} \mathrm{C}$ for 3 days and then subjected to $12 \mathrm{~h}$ of darkness, which synchronized the circadian clock. Bioluminescence from the cells was then automatically monitored under LL (47 $\mu \mathrm{mol} \mathrm{m}{ }^{-2} \sec ^{-1}$ ) with $n$-decanal as the substrate using the photomultiplier tube-based bioluminescence monitoring system as described previously (Aoki et al., 1995). Bioluminescence intensity was measured for $10 \mathrm{sec}$ in darkness at 38 min intervals.

\section{RESULTS AND DISCUSSION}

To address problems in Synechocystis bioluminescence reporter strain $\mathrm{CFC} 2$, we made a new reporter construct, pTS1PdnaK::luxAB(-) (Fig. 1B) in which we seamlessly fused $\mathrm{P}_{\text {dnaK }}$ to $l u x A$ so that the initiation codon of the luxA gene was located at the same position as it was in the 
native dnaK gene. In this construct, 34 extra amino acids that were attached to the amino-terminus of the LuxA protein expressed in strain CFC2 were removed. Removal of this extra sequence would enhance the LuxA activity. The fusion gene was integrated into an intergenic site in the genome where two genes, slr0370 and sll0337, are "tail-to-tail" (targeting site 1). Since integration of exogenous DNA fragments at this site disrupts neither the coding nor the promoter sequences of the two genes, secondary effects due to their disruption were avoided. In addition, we used a short, 805-bp $\mathrm{P}_{d n a K}$ segment that conferred similar rhythmic expression to the 2.2-kbp $\mathrm{P}_{\text {dnaK }}$ segment used in strain CFC2 (Aoki et al., 1995). We transferred pTS1PdnaK::luxAB(-) into Synechocystis cells and assayed the resulting strain, PdnaK ::luxAB(-), for bioluminescence rhythm. The strain showed a robust circadian oscillation that was similar in period length and phase to the oscillation of strain CFC2 (Fig. 2). Amplitude of the oscillation was $11 \%$ higher than that of strain CFC2. The mean bioluminescence intensity over $168 \mathrm{~h}$ was 12 times that of strain CFC2 (Fig. 3).

Next, we tested the effects of transcription direction of

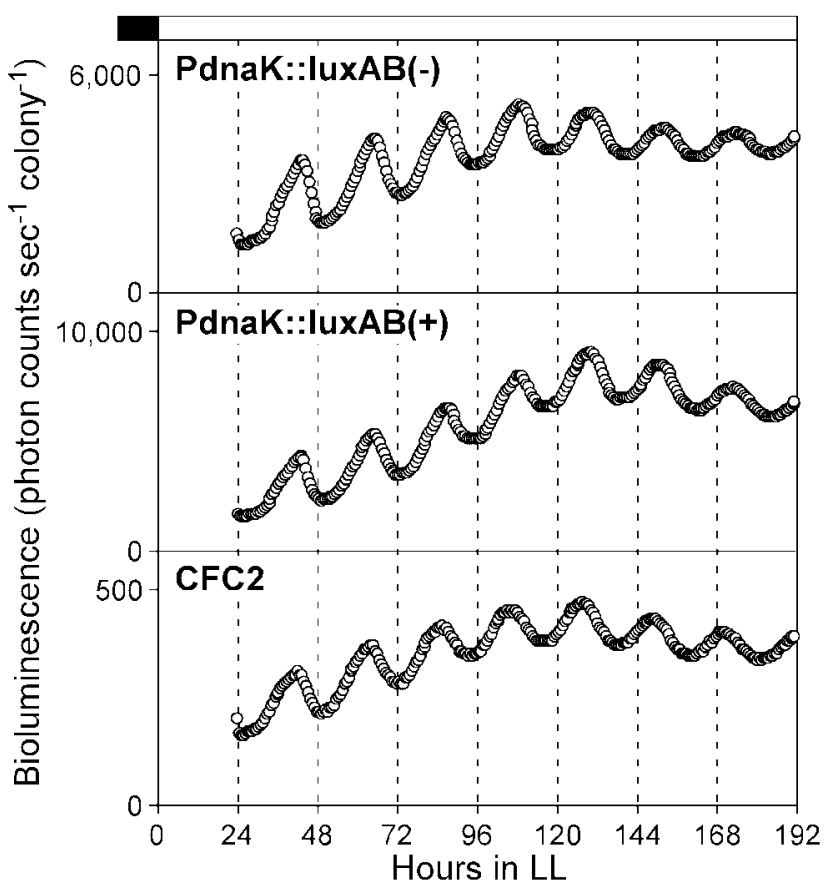

Fig. 2. Bioluminescence rhythms of the reporter strains. Synechocystis cells were subjected to $12 \mathrm{~h}$ of darkness, which synchronized the circadian clock, and were then transferred to LL for monitoring of bioluminescence rhythms. The light regimens are shown by filled (darkness) and open (LL) boxes above the graph. Vertical axis represents the bioluminescence intensity normalized by the number of colonies. Horizontal axis represents time after transfer to LL. Bioluminescence intensity in strain CFC2 is different from that appeared in Aoki et al. (1995) because unit of intensity and bioluminescence monitoring apparatus were different.

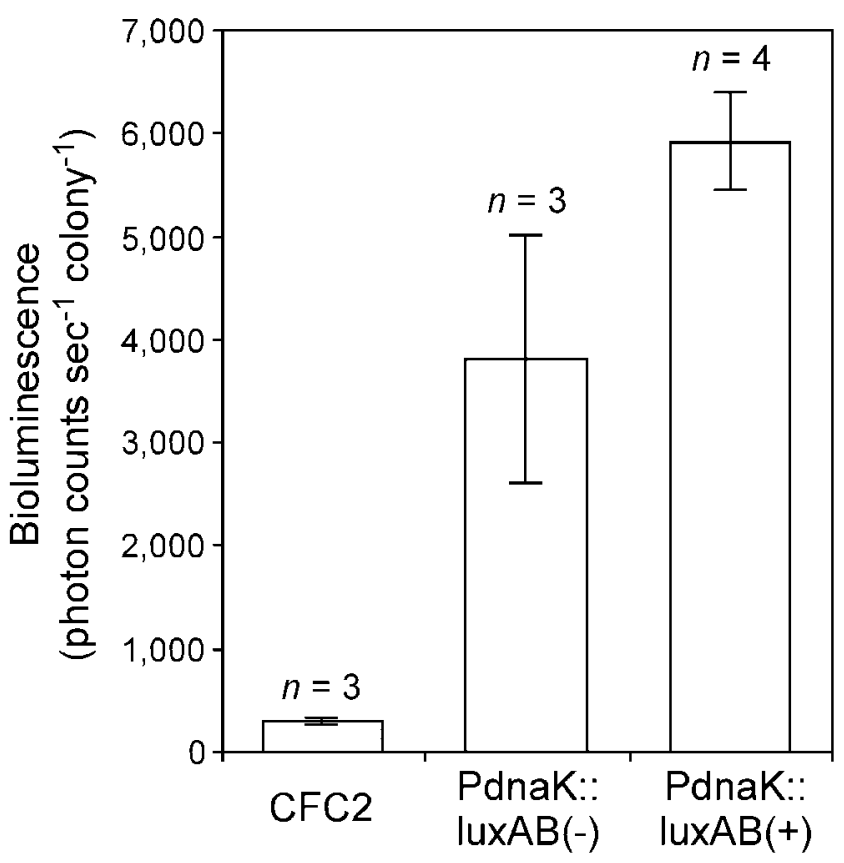

Fig. 3. Bioluminescence intensity of the reporter strains. Boxes represent mean bioluminescence intensity over $168 \mathrm{~h}$ for the indicated number $(n)$ of experiments, normalized by the number of colonies. Bars represent the SD.

the selection-marker $S p^{r}$ gene on bioluminescence intensity. In strain PdnaK::luxAB(-), $S p^{r}$ was located upstream of the $\mathrm{P}_{\text {dnaK }}:$ lux $A B$ fusion gene and transcribed in the same direction (Fig. 1B). We generated a reporter construct, pTS1PdnaK::luxAB(+), in which transcription of the two genes occurred in opposite directions, and integrated it into the same locus as pTS1PdnaK::luxAB(-) (Fig. 1B). Fig. 2 shows the bioluminescence rhythm of the resulting strain PdnaK::luxAB(+). This strain's rhythmic pattern was similar to that of strain PdnaK ::luxAB(-), but the mean bioluminescence intensity was $60 \%$ greater than that of strain PdnaK::luxAB(-) (Fig. 3). Amplitude of the rhythm was $10 \%$ higher than that of strain CFC2. In strain PdnaK::luxAB(-), movement of RNA polymerase on $S p^{r}$ toward the $\mathrm{P}_{\text {dnaK }}:$ lux $A B$ fusion gene would cause positive supercoiling of $d n a K$ promoter region. This would negatively affect transcription of the $\mathrm{P}_{\text {dnaK }}:$ lux $A B$. Finally, the bioluminescence intensity of strain PdnaK::luxAB(+) was 19 times that of strain $\mathrm{CFC} 2$ while the period length and phase of the rhythm were unaffected. As a result, the improved bioluminescence intensity of strain PdnaK::luxAB(+) was comparable with that of the Synechococcus psbAI reporter strain (Kondo et al., 1993).

In this study, we elucidated the critical factors for generating reporter strains with high bioluminescence intensity: (i) seamless fusion of the $\mathrm{P}_{d n a K}$ sequence to the luxA gene, and (ii) anti-parallel positioning of the fusion gene and the $S p^{r}$ selection marker gene. These will be useful 
for analyzing bioluminescence rhythms of genes with low promoter activity. Furthermore, the new constructs should facilitate analysis of a large number of promoters because the promoter fragment contained in the constructs can easily be replaced by digestion with unique AflII-NdeI sites (Fig. 1B). Recently, we developed a high-throughput bioluminescence monitoring apparatus using 96-well microplates (Okamoto et al., 2004). Thus, the new reporter constructs should make a significant contribution to genome-wide analysis of circadian gene expression in Synechocystis.

We thank Dr. Thomas O. Baldwin (Texas A\&M University) for the gift of plasmid pLAV1, and Dr. Miriam Bloom (SciWrite Biomedical Writing \& Editing Services) for professional editing. This work was supported by grants from the Japanese Ministry of Education, Science and Culture (MEXT), 'Program for Promotion of Basic Research Activities for Innovative Biosciences' (PROBRAIN) promoted by BRAIN, 'Research for the Future Novel Gene Function Involved in Higher-Order Regulation of Nutrition-Storage in Plants' promoted by the Japan Society for the Promotion of Science, 'Ground-based Research Announcement for Space Utilization' promoted by the Japan Space Forum, 'National Project on Protein Structural and Functional Analyses' promoted by MEXT, and 'Joint-Project for Leading Science and Technology' promoted by the Aichi Science and Technology Foundation to M.I. Division of Biological Science, Graduate School of Science, Nagoya University was supported by a 21 st century COE grant from MEXT.

\section{Added in proofs:}

We developed an integrated bioluminescence rhythm-analyzing program (RAP) with a user-friendly graphical interface [Okamoto, K., Onai, K., and Ishiura, M (2005) RAP, an integrated program for monitoring bioluminescence and analyzing circadian rhythms in real time. Anal Biochem. in press].

\section{REFERENCES}

Anderson, S. L., and McIntosh, L. (1991) Light-activated heterotrophic growth of the cyanobacterium Synechocystis sp. strain PCC 6803: a blue-light-requiring process. J. Bacteriol. 173, 2761-2767.

Aoki, S., Kondo, T., and Ishiura, M. (1995) Circadian expression of the dnaK gene in the cyanobacterium Synechocystis sp. strain PCC 6803. J. Bacteriol. 177, 5606-5611.

Aoki, S., Kondo, T., Wada, H., and Ishiura, M. (1997) Circadian rhythm of the cyanobacterium Synechocystis sp. strain PCC 6803 in the dark. J. Bacteriol. 179, 5751-5755.

Baldwin, T. O., Berends, T., Bunch, T. A., Holzman, T. F., Rausch, S. K., Shamansky, L., Treat, M. L., and Ziegler, M. M. (1984) Cloning of the luciferase structural genes from
Vibrio harveyi and expression of bioluminescence in Escherichia coli. Biochemistry 23, 3663-3667.

Ishiura, M., Kutsuna, S., Aoki, S., Iwasaki, H., Andersson, C. R., Tanabe, A., Golden, S. S., Johnson, C. H., and Kondo, T. (1998) Expression of a gene cluster kaiABC as a circadian feedback process in cyanobacteria. Science 281, 15191523.

Kaneko, T., Sato, S., Kotani, H., Tanaka, A., Asamizu, E., Nakamura, Y., Miyajima, N., Hirosawa, M., Sugiura, M., Sasamoto, S., Kimura, T., Hosouchi, T., Matsuno, A., Muraki, A., Nakazaki, N., Naruo, K., Okumura, S., Shimpo, S., Takeuchi, C., Wada, T., Watanabe, A., Yamada, M., Yasuda, M., and Tabata, S. (1996) Sequence analysis of the genome of the unicellular cyanobacterium Synechocystis sp. strain PCC6803. II. Sequence determination of the entire genome and assignment of potential protein-coding regions. DNA Res. 30, 109-136.

Kondo, T., Strayer, C. A., Kulkarni, R. D., Taylor, W., Ishiura, M., Golden, S. S., and Johnson, C. H. (1993) Circadian rhythms in prokaryotes: luciferase as a reporter of circadian gene expression in cyanobacteria. Proc. Natl. Acad. Sci. USA 90, 5672-5676.

Kucho, K., Okamoto, K., Tsuchiya, Y., Nomura, S., Nango, M., Kanehisa, M., and Ishiura, M. (2004) Global analysis of circadian expression in the cyanobacterium Synechocystis sp. strain PCC 6803. J. Bacteriol. in press.

Millar, A. J., Short, S. R., Chua, N. H., and Kay, S. A. (1992) A novel circadian phenotype based on firefly luciferase expression in transgenic plants. Plant Cell 4, 1075-1087.

Okamoto, K., Onai, K., Ezaki, N., Ofuchi, T., and Ishiura, M. (2004) An automated apparatus for the real-time monitoring of bioluminescence in plants. Anal. Biochem. in press.

Onai, K., Morishita, M., Itoh, S., Okamoto, K., and Ishiura, M. (2004) Circadian rhythms in the thermophilic cyanobacterium Thermosynechococcus elongatus: compensation of period length over a wide temperature range. J. Bacteriol. 186, 4972-4977.

Orosz, A., Boros, I., and Venetianer, P. (1991) Analysis of the complex transcription termination region of the Escherichia coli $r r n B$ gene. Eur. J. Biochem. 201, 653-659.

Prentki, P., and Krisch, H. M. (1984) In vitro insertional mutagenesis with a selectable DNA fragment. Gene 29, 303-313.

Rippka, R., Deruelles, J., Waterbury, J. B., Herdman, M., and Stanier, R. Y. (1979) Generic assignments, strain histories, and properties of pure cultures of cyanobacteria. J. Gen. Microbiol. 111, 1-61.

Sambrook, J., Fritsch, E. F., and Maniatis, T. (2001) Molecular Cloning, third edition. Cold Spring Harbor Laboratory Press, New York.

Williams, J. G. K. (1988) Construction of specific mutations in photosystem II photosynthetic reaction center by genetic engineering methods in Synechocystis 6803. Methods Enzymol. 167, 766-778. 